\title{
Feedback for the Seventh International AIDS Conference, Florence 1991
}

\section{Introduction}

Following the Seventh International AIDS Conference in Florence in June 1991 a feedback day for Genitourinary Medicine Physicians was held at the Royal Zoological Society, London. This is a report from the feedback. The abstracts are cited in the text using the code number from the published abstract books. A separate conference entitled Neurological and Neuropsychological Complications of HIV Infection was held in Padua, Italy in the week before the main AIDS conference, and one of the contributors has included abstracts from Padua.

\section{Virology-Malcolm Semple \\ Pathogenesis}

Data were presented at the conference which suggested that spermatozoa were a vector and cofactor for the transmission of HIV. Rao (MC3094) presented electron micrographs showing HIV bound to the surface membrane of sperm. Schofield (WC50) demonstrated that sperm bind to somatic cells which are abundant in the female reproductive tract. Some of these cells express the HIV receptor molecule CD4 and the binding of sperm to these cells stimulated cell division. The authors concluded that because cellular infection and viral replication are sensitive to cellular activation, sperm may contribute to the conditions which result in HIV infection by the sexual route.

William Haseltine described how HIV can gain access to the body via mucosal surfaces of the genitourinary and gastrointestinal tract, and infect T-cells in lymph glands, without mucosal trauma and without the virus entering the blood (fig 1). Haseltine presented electron micrographs describing HIV being transported across mucosal $M$-cells by cytosis and then infecting tissue dendritic cells, which are phagocytic, CD4 positive antigen processing and presenting cells which are more susceptible to HIV infection. They produce more than 50 times as much virus and are more resilient to damage by the virus than peripheral blood leukocytes (PBLs) (WA70, TUB27). Langerhans cells of the skin are the only epidermal cells which can be infected by HIV (ThA18). Langerhans cells are also dendritic, antigen presenting cells and share the same lineage as the tissue dendritic cells of the mucosa. Both cell types migrate to lymph glands where they develop into interdigitating cells, that is, antigen presenting cells found in contact with T-helper cells. Graziosi demonstrated that 10 times as many $T$ cells were infected in patients' lymph nodes than in their

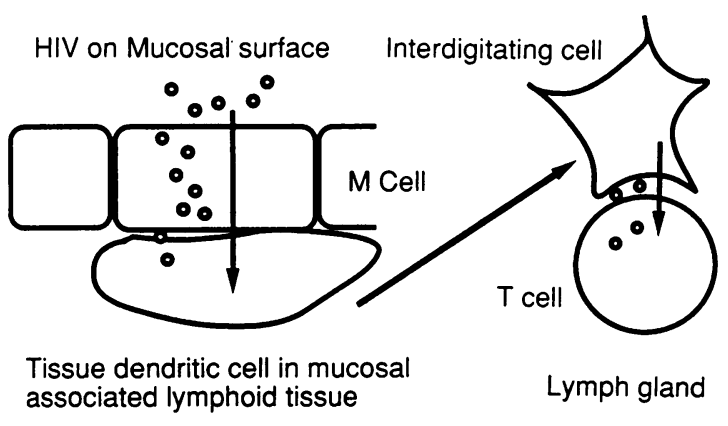

Figure 1 Model for the mechanism of HIV infection. HIV is transported by cytosis across the mucosa via M cells. HIV then infects tissue dendritic (TD) cells, which are resistant to damage and produce large amounts of virus. TD cells migrate to lymph gland where they develop into interdigitating cells and infect $T$ cells.

peripheral circulation and concluded that the lymphoid tissue functions as a major reservoir for HIV infection (TUB28). Knight demonstrated that antigen presentation became abnormal in dendritic cells when they were infected in vitro with HIV (TUB29). The sum of these data has implications for the development of vaccines and goes some way in explaining the failure of both cellular and humoral immune responses in infected people.

Schultz (ThA1) and Sattentau (ThA3) presented data demonstrating that enzymatic cleavage of the V3 loop of gp160 is required for HIV to infect cells and further, Sattentau demonstrated that CD4 induces this specific cleavage.

At the genetic level, Stevenson (MA6) and Goudsmit (MA8) described how in asymptomatic people the proviral genome remains circular in the cytoplasm of latent cells and so is not available for translation by the cell's nuclear machinery, but in symptomatic patients where the greater proportion of these cells is activated, the proviral genome is found in the nucleus in the linear form, therefore transcription, translation, expression and viral production can occur. Goudsmit went on to describe very elegant work regarding diversity of the hypervariable V3 loop of gp160, the principal neutralising domain of HIV. He described how in the asymptomatic phase of infection variability was driven by an active immune system and viraemia was suppressed. As immune function deteriorated and CD4 cell count dropped, diversity was lost and viraemia increased, that is, a few variants escaped immune clearance, became dominant and increased in number. This coincided with the emergence of 


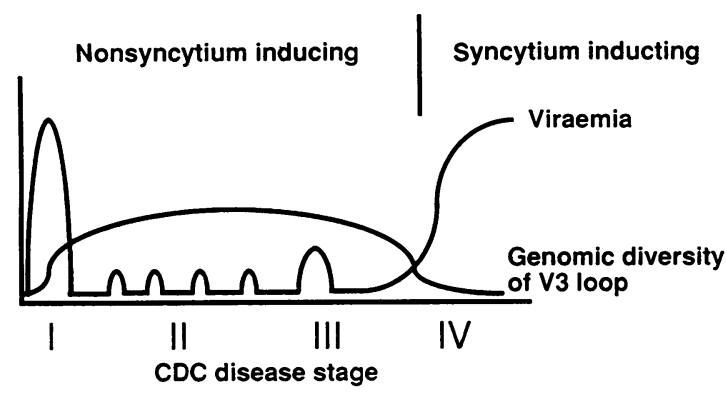

Figure 2 Levels of genomic diversity of the V3 loop of gp120 against progression of HIV disease.

variants capable of forming multinucleated syncytia in vitro. He also described a large spike of viraemia at the time of seroconversion (fig 2). This has implications for maternal-fetal transmission where an association between maternal seroconversion and vertical transmission was described (WC3247).

\section{Vaccines}

On the whole there was little encouraging news regarding vaccines. The success derived using the simian immunodeficiency virus (SIV) and SIV vaccines (MA24) was tempered by growing evidence that the differences between the properties of the immunogenic regions of SIV (whole native gp120) and HIV (V3 region) were so great that direct extrapolations to a human vaccine can not be made (ThA14). Furthermore the model described by Haseltine for the transport of HIV to the lymphoid system and the establishment there of a reservoir of cellular infection means that to be effective a vaccine will be required to stimulate a cell mediated response and a humoral response. Such a vaccine will probably require a live attenuated vector. Gardner described a whole killed SIV vaccine that protected macaques from an intravenous challenge of live virus, but did not provide protection from a mucosal challenge of live virus (WA1120).

McCutchan presented data describing the geographical diversity of HIV and concluded that a vaccine effective in one country or city would not necessarily be protective in another (ThA32).

There was optimism from Dani Bolognesi that within three years large scale efficacy trials for prophylaxis or immunotherapy based upon vaccine regimes would start where the initial dose is a live attenuated vaccinia gp160 recombinant and the booster is a peptide subunit of gp160. However, the administration of live vaccinia recombinants to immunocompromised patients is absolutely contraindicated because of the risk of generalised vaccinia (Lancet, 1991;337:1034-5).

\section{Drug resistance}

The emergence of drug resistant strains in patients who had received zidovudine and dideoxyinosine (ddI) was reported by several investigators. Boucher reported that in five patients, the emergence of mutations in the pol gene associated with zidovudine resistant in vitro occurred in an orderly sequence. Firstly at codons 70 and 215 conferring partial resistance and then later at positions 67,219 and 41 conferring a high level of resistance. A high level of resistance was not required for disease progression and partial resistance (mutations at codon 215) persisted after zidovudine therapy ceased (TUB89).

Boardhurst described point mutations in primary isolates from 28 individuals who had been treated with zidovudine, which were resistant in vitro (TUB87). The areas where mutations occurred were centred around the pol codon regions $65-75$ and 185-220, believed to code for functional domains of reverse transcriptase. Drug resistance emerged in less than three months in some patients and CD4 cell counts decreased as mutations emerged.

While these data demonstrated the emergence of resistant strains, they did not address their clinical significance. Semple et al presented a strategy for the direct, independent, rescue, detection and quantification of HIV-1 RNA from the stored sera of infected patients using reverse transcribed nested polymerase chain reaction (RNA PCR) and applications for these methods (ThA17). They demonstrated an increase in viraemia as HIV disease progressed, a modification of viraemia during antiviral therapy and the emergence of variants with point mutations associated with zidovudine resistance. These zidovudine resistant strains were found in greater abundance in serum than in peripheral blood lymphocytes (fig 3 ). Significant levels of virus were detected in patients with CDC stages II, III and IV, and there was a tenfold increase in geometric mean viraemia between asymptomatic and symptomatic patients. These data indicate that viraemia as measured by this method would be a good marker for the efficacy of antiretroviral therapy and the emergence of resistant variants.

Cross-resistance between dideoxyinosine (ddI) and zidovudine was described by Bach (TUB91). Six patients who had previously been treated with zidovudine and in whom resistance was demonstrated in vitro were treated with ddI for 12 to 15 months. One of the six was initially found to carry cross resistant virus and died shortly after starting ddI therapy. One could not tolerate ddI and left the trial. The remaining four responded to ddI therapy. Zidovudine resistance partially decreased as ddI resistance emerged, however the mutation at codon 215 was maintained. Bach postulated that the mutation at 215 could confer partial cross-resistance between ddI and zidovudine. It should be noted that these were end stage patients and that it would appear that resistance is slower to develop when therapy is 


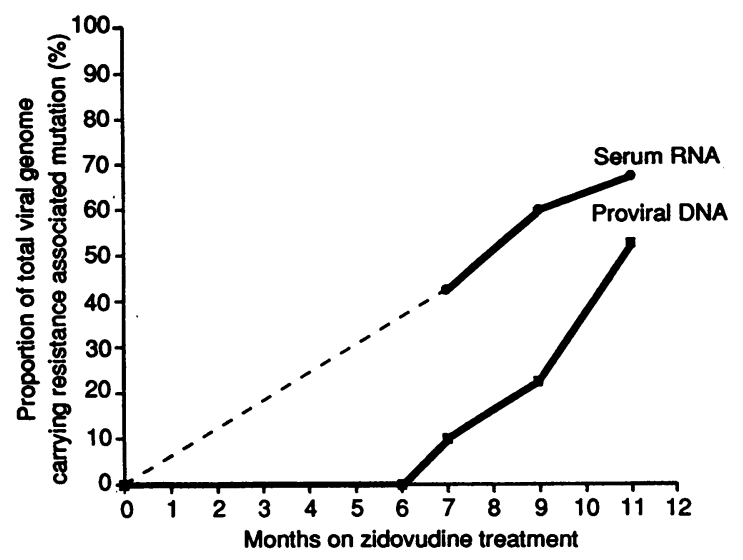

Figure 3 Emergence of $H I V-1$ strains with resistance associated mutation at codon 70 in serum and peripheral blood leucocytes in a patient treated with zidovudine.

initiated during the asymptomatic disease stages, possibly because the viral load and therefore the chance of a resistant variant emerging are both less.

\section{Epidemiology-Anne Johnson}

Close to 800 abstracts were presented on the epidemiology of HIV worldwide. All provided evidence that an increase in both HIV prevalence and cases of AIDS can be expected to the year 2000 . Some new areas of interest included patterns of spread worldwide and problems of measuring the incidence of HIV infection.

\section{Worldwide Spread}

Dr James Chin from the World Health Organisation (WHO) provided a keynote overview of the current and projected future magnitude of the epidemic. As of 1991, over 9 million HIV infections are believed to have occurred worldwide. Of these, an estimated 1.5 million have occurred in the western world and 6 million in sub-Saharan Africa. WHO project that by 1995 over 15 million infections will have occurred worldwide, bringing the regional totals to 10 million in sub-Saharan Africa, 2 million in the western world, 2 million in Latin America, and 2.5 million in south and southeast Asia. Because the median incubation period for HIV infection is in the order of 10 years, projections for the total number of AIDS cases are largely based on the number of people currently infected. By 1991, a total of 1 million cases of AIDS had occurred worldwide and it is estimated that 10 million will have occurred by the year 2000 . By the year 2000, the number of new cases occurring annually will reach more than 1 million. Of the latter, more than $50 \%$ are projected to occur in Africa.

Wide coverage was given in the press to the rapid increase in HIV and AIDS cases in African countries. It is, however, important to remember that projections for the western world indicate that a further half million infections with HIV are projected to occur by 1995 over and above the 1.5 million which have occurred since the start of the epidemic. Thus the number of new AIDS cases annually in the developed world is projected to increase into the mid-1990s and then remain high to the year 2000 .

It is now clear that worldwide, heterosexual spread of HIV is increasingly the predominant mode of transmission. Of infections occurring worldwide up to $1991,60 \%-70 \%$ are estimated to have occurred by vaginal intercourse, $5-10 \%$ by anal intercourse, 5$10 \%$ through injecting drug use, $3-5 \%$ by blood transfusion, and 5-10\% through perinatal transmission.

\section{Incidence/Prevalence Studies}

Many abstracts showed that there was evidence of high seroprevalence both in higher risk populations and the general population in Africa (WC101, WC3251, WC3262, WC3265). In Latin America there is evidence of spread through injecting drug use, male homosexual intercourse and increasingly through heterosexual intercourse (MC3267, MC3270, MC3008, MC3010, MC3015). In south and south-east Asia there is now evidence of increasing spread of HIV, particularly through heterosexual transmission, but also through injecting drug use (MC3246, MC1, MC3097, MC3307, MC3086). Amongst samples of injecting drug users in Thailand (MC3246), the median seroprevalence has now risen to over $30 \%$. Amongst female sex workers, the median seroprevalence rose from 3.5\% in June 1989 to $9.3 \%$ in June 1990, with infection rates of up to $67 \%$ in some specific areas, mirroring the rapid spread of HIV infection amongst female sex workers in some sub-Saharan African cities in the mid-1980s. Evidence of heterosexually acquired infection is becoming increasingly evident amongst male STD clinic attenders, with a median seroprevalence of $2 \cdot 5 \%$, but estimates of up to $24 \%$ in some selected areas.

In the developed world, several areas of concern emerge. These include the emergence of infection amongst adolescents and students in the USA (WC338, WC3128, WC268). Evidence of increasing heterosexual spread in the western world was highlighted (WC102). In certain sectors of the population in the USA, high rates of infection $(8.4 \%$ in women, and $5.8 \%$ in men) were reported in heterosexual attenders at a New York STD clinic, excluding injecting drug users and gay men. Age, crack and syphilis, together with a history of IDU partners were associated with infection. Thus evidence accumulates for the emergence of heterosexual spread in deprived inner-city areas, particularly amongst ethnic minorities (MC3093, WC45, WC99). 
A major area of concern was evidence of the continuing high incidence of HIV infection amongst new injecting drug users and young gay men. These presentations highlighted difficulties in interpreting the rate of new infections from serosurveillance efforts based on anonymous unlinked prevalence studies.

As cohorts age and the death rate from AIDS increases, stable prevalence estimates may mask a high incidence of HIV infection in those becoming sexually active and those embarking on their injecting drug using careers. Thus, for example, Des Jarlais (MC1) reported a stable prevalence in injecting drug users in New York City for six years of approximately $45 \%$. Despite this, it is estimated that the current incidence of HIV infection in New York amongst injecting drug users is 3-4 per 100 person years. Seroconversions are concentrated in new injectors and are occurring despite reports of decreased risk behaviours in developed countries. Similar incidence rates were reported in IDUs in Italy. The findings reflect the fact that once prevalence becomes sufficiently high, very considerable changes in behaviour are required in order to eliminate new infections.

Similarly amongst gay men, there is some evidence that previous data based on cohort studies of gay men have underestimated incidence. For example, in the Vancouver lymphadenopathy study (WC95), a disproportionate drop-out rate was reported by those at highest risk at the start of the study. Thus as cohorts are followed over long periods of time, they may become decreasingly representative of the gay community, owing to a number of biases. These include selection bias (as such studies involve committed volunteers), and an ageing process, both of which may lead to an underestimate of incidence. Two alarming pieces of evidence indicated that HIV is now being passed from one generation to another. Amongst gay men attending STD clinics in San Francisco and the Bay area, an unlinked anonymous testing study showed that amongst 15-19 year old homosexual men who became sexually active after the start of major prevention campaigns amongst gay men, over $15 \%$ are now infected. Similarly in the UK in an opportunistic cohort reported by Communicable Disease Surveillance Centre (WC3000), it was estimated that amongst gay men in London with a previous negative test presenting for re-testing, the incidence of HIV infection was 4.6 per 100 person years, and 2.29 per 100 person years in those outside London. Nevertheless, clinic samples may overestimate prevalence, and in data from Project Sigma (WC3020) prevalence of HIV was found to exceed $18 \%$ in gay men from a clinic sample, but to be only $1.4 \%$ in a non-clinic sample.

In summary, there is evidence that HIV has now reached all corners of the world, and in some, infection is increasing rapidly, notably through heterosexual transmission, particularly in parts of the developing world. Control of the epidemic still relies, and will rely in the foreseeable future on behaviour change. Further efforts to introduce and evaluate different strategies continue to be urgently needed, for the greater the magnitude of the increasing pandemic the more difficult control becomes.

\section{HIV and AIDS in women-Richard Keenlyside Epidemiological Trends}

Investigators from the Communicable Disease Surveillance Centre reported on the epidemiology of HIV-1 infection in women in the UK (WC3120). AIDS and HIV infection in women in the UK are increasing at a faster rate than in men. Although the proportion of women among known infected adults was low (4\% of AIDS cases and $9 \%$ of HIV infections December 1990) the 619 infections in women reported in 1988 were $60 \%$ greater than 379 reported between 1985-87. Also between 1985 and 1990 the ratio of females to males has declined from 1:50 to $1: 15$ for AIDS cases and from 1:48 to $1: 7$ for HIV infections. During the same period the percent of new HIV infections attributable to heterosexual transmission has increased from $43 \%$ to $70 \%$.

Berkleman reported from the Centers for Disease Control (CDC) (WC102) on the increasing role of heterosexual transmission in the United State. Fifty five percent of all cases in women reported in USA $(15,493$ cases) have been reported in the past two years. Although $51 \%$ of cases reported so far have occurred in intravenous drug users heterosexual cases have the highest rate of increase. In $1990-31 \%$ of cases in women were attributable to heterosexual contact.

In the USA, 60,000 men and women have been tested annually since 1987 for entry into a job training programme for poor undereducated unemployed adolescents: Among these, the prevalence of HIV increased with age, and among 21 year old blacks of both sexes the prevalence was over $1 \%$. Since screening began the sex ratio has changed and now females have a prevalence 1.41 times that of males (WC3268). In Quebec Canada (MC3259) $9.6 \%$ of cases have been in women and heterosexual exposure is their most common exposure factor.

Trends in the AIDS epidemic and patterns of AIDS defining illness were reported in 95 cases of AIDS in Scandinavian women (MC3171). Fifty three percent were heterosexually acquired, $26 \%$ through transfusion of blood or blood products and $19 \%$ through intravenous drug use. The proportion heterosexually acquired has increased over time from $21 \%$ before 1988 to $77 \%$ in 1990 . There is increasing evidence of the changing patterns of HIV infection in the Caribbean and South America. In Rio de Janeiro by December $199011.5 \%$ of all cases were in women 
(MD4238) and the ratio of females to males had decreased from 9:1 in 1987 to 6:1 in 1989. A similar pattern was seen in Martinique (MD4239) where the sex ratio has fallen from 2.7 to 1.4 and $85 \%$ of female and $51 \%$ of male cases were heterosexually acquired. In Africa, among 1000 female prostitutes studied prospectively in Senegal the rates of infection for HIV-1 have increased 8 fold in 6 years but the rates for HIV-2 have remained relatively stable (MC41).

\section{Clinical Features}

There were several reports of the clinical features of HIV infection in women with comparisons of the features of illness in men. Investigators claimed to identify differences between the sexes but the findings were not very consistent. Among women in Rhode Island, the most common AIDS defining diagnoses were oesophageal candidiasis (33\%); chronic herpes simplex (25\%) and PCP pneumonia $(22 \%)$ (MC3000). In addition high grade squamous epithelial lesions occurred frequently.

In a study comparing the sex differences in the prevalence AIDS indicative diagnoses at the time of reporting to the CDC oesophageal candidiasis and herpes simplex infection were also found to be more common in women with AIDS (MC3210).

In a cohort of 2639 persons with HIV infection seen in Atlanta, women were more likely than men to have oesophageal candidiasis, atypical mycobacterial infection, and wasting syndrome (MC3115). Among 117 sero positive heterosexuals in New England, HIV positive women and men had different clinical illnesses and symptoms from each other and other reported groups. Women had a higher prevalence of anaemia, constitutional symptoms, yeast infections and warts (MC3117).

However, in a study of hospital cases in New York City, AIDS defining illnesses were similar in men and women, apart from Kaposi's Sarcoma which was more common in homosexual males (MC3192) and a study by the CDC of 465 patients in 6 states also failed to confirm these differences (MD4253).

It appears that, for some conditions, women differ from men in their patterns of both AIDS defining diagnoses and non AIDS defining conditions but this was not shown in all the studies. The studies were difficult to compare because they differed with respect to sample size, methodologies used, length of follow up, the mixture of risk factors and the different stages of progression in their subjects. However, they highlighted the need for more rigorous research to better describe the disease in women and especially to study the correlation between $\mathrm{CD} 4$ counts and disease presentation.

\section{Survival}

There were several studies to investigate the association of gender with survival after the AIDS diagnosis. In a Harvard University study of CDC data examining the relative hazards of death for females compared with males adjusted for race, age, region and initial diagnosis women had a small but consistently raised mortality risk relative to men in three of the four risk groups (MC3135). Among 125 adult women in San Francisco, survival by year of diagnosis was found to be significantly shorter in women than in men (MC3122). It has increased over time in men but not in women. Older age, infection by transfusion was associated with decreased survival. Among those not using AZT women had a shorter survival than men but there was no difference in survival in those treated with AZT. In a study of the case records of 1254 patients in Atlanta age, race and intravenous drug use were not associated with decreased survival (MC3175). In a proportional hazards analysis of the data a lower CD4 count, AIDS diagnosis, treatment and female gender each seemed to be associated with decreased survival and therapy was protective. However, when other variables were considered in the model only AIDS diagnosis and $\mathrm{CD} 4$ count predicted poor survival and being female did not.

\section{Pregnancy}

Studies from Italy (WC3235) and Zaire (MC3149) on the impact of pregnancy on the cumulative progression of disease and trends in the deterioration of immune function were inconclusive largely because of the small numbers studied and too brief follow up of the subjects.

Johnson and colleagues in Edinburgh continue to study and report on their population based cohort of pregnant women infected with HIV and matched controls (WC3239). Multifactorial analysis indicated that HIV itself is associated with low birth weight, and similar findings were reported from a study of HIV-1 and HIV-2 infected women in the Ivory Coast (MC3053).

\section{Screening and seroprevalence of HIV in antenatal patients}

The results of screening antenatal patients for HIV infection were reported from several countries. The highest rates were reported from Central Africa where $28 \%$ of women attending a prenatal clinic in Uganda (WC3262) and 5.5\% of low risk patients in an obstetric ward in Kenya (WC3263) were seropositive. In both countries the seroprevalence rates continue to increase, especially in the rural populations (WC3283).

In the USA over 3 million women and their offspring have now been screened through testing of newborn dried blood specimens (WC34), and approximately 1.5 per 1000 women delivering in the USA in 1990 were infected resulting in $1,200-1,500$ 
prenatal infections per year. Seroprevalence has not changed in 14 states with 2nd and 3rd serosurveys. So far more than 5000 children have been perinatally infected in the US since 1980 and only 2327 had been reported with AIDS by January 1991. More than half have not yet progressed to AIDS and over 1000 new cases are expected in 1992.

In Milan $0.36 \%$ of all pregnant women screened were seropositive (WC3242). Elsewhere in Italy, in Latium (WC3254) and Liguria (WC3275) the prevalence among antenatal patients was $0.36 \%$ and $0.28 \%$ respectively. There was also an alarmingly high rate $(1.6 \%)$ found among 763 women tested consecutively in Barcelona, Spain (WC3281). In Sweden (WC3278) and Norway (WC3279) routine national antenatal HIV-1 screening programmes have been in place since 1987 and these have had high acceptance rates (90-95\%). After three years of testing the seroprevalence rates were $0.001 \%$ in 324,256 women tested in Sweden and $0.008 \%$ in 276,852 women in Norway.

\section{Perinatal transmission}

Studies of the maternal factors influencing perinatal transmission from Edinburgh (WC3237) and Italy (WC3277) revealed that there is a greater risk of transmission if the child in born to an infected mother within 1 year of the mothers seroconversion, if the mother has a low CD4 count, is p24 antigen positive or has advanced HIV disease. Also bearing an infected child is associated with a poorer prognosis in the mother even if she is asymptomatic at delivery. There was, however, a very low rate of transmission among the patients $(6.4 \%)$ in the Edinburgh study which was not readily explained.

A study from Kenya (MC3062) showed that there was a significant correlation between maternal viral burdens measured by quantitative PCR and transmission to the child. HIV was identified in cord blood in only $10 \%$ of children, compared with $20-40 \%$ at 6-9 months indicating that perhaps perinatal and post natal transmission may be more common than in utero.

Other clues that this might be the case came from an interesting cohort study in Rwanda (WC33) that looked at transmission events from mother to infant when the mother became infected with HIV after delivery. Among 219 mother-infant pairs who were sero negative at delivery, $16(7 \cdot 3 \%)$ of the mothers became infected during the follow up and the infection was apparently passed on to $9(56 \%)$ of their children. It was also found that early infection of the mother post partum was asssociated with higher risk of transmission to the child. The authors suggested that colostrum or breast milk could be efficient routes for transmission. This has now to be considered as a possibility since Ruff and co-workers in the USA (MC3009) have detected HIV by PCR in breast milk in $71 \%$ of women infected with HIV. In their study colostrum was more likely to be positive than later breast milk. A study from Italy (MC3024) supports these findings. These disturbing findings do not yet indicate a need for changes in policy for breast feeding but indicate that the issue needs to be pursued further.

\section{Progression of HIV infection to AIDS and} potential cofactors-Andrew Phillips

Several studies suggest that treatment of AIDS-free HIV infected patients, primarily with zidovudine and PCP prophylaxis, has reduced the rate of progression to AIDS (eg MC3187, WC41). For example, in a cohort of haemophiliacs there was, before November 1988 when treatment for asymptomatic patients began, an average of one AIDS case per 3.1 years of follow-up among patients in whom the CD4 lymphocyte count had fallen below 200 cells $/ \mathrm{mm}^{3}$. After November 1988 this changed to one case per 5.3 years (MC3187). Thus it appears that treatment has had some effect on the rate of progression to AIDS. Those who have acquired the infection more recently may then have a better prognosis than those who seroconverted 5 or more years ago. This means that estimates from cohorts of HIV infected patients who have been followed for many years are likely to overestimate somewhat the rate at which future patients will progress to disease, assuming these future patients receive the available treatment.

Nonetheless, it is unfortunately already clear that the effect of existing treatments on the natural history of the infection, although real, is rather limited so estimates from the long follow-up cohort studies are still very pertinent. Among homosexual/bisexual men in the San Francisco City Clinic cohort the estimated percentage of patients progressing to AIDS by 12 years from seroconversion is $62 \%$ (WC42). This contrasts with a figure of $44 \%$ (95\% confidence limits $29 \%-59 \%$ ) for UK haemophiliacs at 11 years from seroconversion (MC3187).

The difference between these estimated progression rates could be explained by the fact that the former group tended to be somewhat older than the latter. It has been consistently reported in the literature that age is a so-called "co-factor" which influences the rate of progression to AIDS-with the exception of young children, older patients tend to progress more rapidly than younger ones. This was confirmed in several reports (MC45, MC3187, MC3131, TUC43, TUC50, MC3148, MC3204) and, surprisingly, it remains the only co-factor that has been consistently identified: any differences that there are in progression rates according to sex, race or transmission category in the West can apparently be explained in terms of age differences (TUC43, MC3148, MC3178). However, given the relatively 
small numbers of women and non-caucasians followed this is certainly not conclusively established. In Africa, rates of progression to AIDS appear to be much greater than in the West. A study of Nairobi prostitutes found that $50 \%$ of women progress to AIDS within 4 years of infection (TUC103).

Several other possible co-factors were reported to be associated with more rapid progression including the presence of other sexually transmitted diseases (MC95, TUC103, MC3110) and previous CMV infection (MC3187). Abstinence from heroin was reported to be associated with a lower rate of progression of AIDS in Italian intravenous drug users (MC3180). Data were also presented concerning another postulated viral co-factor. Patients with human herpes virus 6 (HHV-6) were found to experience a more rapid rate of decline in CD4 lymphocyte count than those without (MC3164). Several studies, from Spain (MC3309), France (MC3188) and the United States (MC3153, WA1227), looked at HLA type, which others have previously found to relate to progression rate, but no consistent pattern concerning such a genetic cofactor is yet emerging.

\section{Markers of progression}

Like co-factors, these are factors that are associated with the rate of progression to AIDS. They differ from co-factors in that they reflect the course of the infection rather than actually helping to determine it. These markers tend to fall into four broad categories. (1) Markers of the amount and pathogenicity of virus present. The presence of $\mathrm{p} 24$ antigenaemia has been shown to relate to the rate of progression to AIDS and this was confirmed in several reports (MC3129, MC3154, TUC39). One of these reports (TUC39) suggested that the increased progression rate in p24 antigenaemic patients can be explained by a tendency for the CD4 count to decline more rapidly in these patients. New tests which measure p24 antigen after dissociating immune complexes may provide a more sensitive measure of viral load than the presently used p24 antigen assays (MB34, MB38). Two reports from Amsterdam (TUC101, WA80) indicated that the presence of syncytium inducing viral isolates and of isolates that productively infect the MT2 cell line are associated with more rapid progression to AIDS. (2) The specific immune response to HIV. Little new epidemiological evidence was presented concerning the prognostic power of markers of aspects of the HIV specific immune response. However, the titre of p24 antibodies, was again reported to be of significance (MC3194). One study from the United States found racial differences in humoral immunity with higher titres of anti-p24 in blacks than Caucasians (MC3258).

(3) General activation of the immune system. Serum levels of beta2 microglobulin, neopterin, IgA, soluble $\mathrm{I} 2$ receptors, soluble $\mathrm{CD} 8$ receptors and interferon were all again shown to be significantly associated with the rate of progression to AIDS (TUC99, WA1137, WB2426, WB2436, WB2444, THC57, MC3129, MC3142). Of these markers, beta 2 microglobulin and neopterin appear to be the most consistently and strongly predictive. It has been suggested that the increase in numbers of CD8 lymphocytes often seen in HIV infection also reflects the general immune activation but, interestingly, this was shown by two studies to be associated with a slower rate of progression to AIDS (WB2438, WB2440). One new prognostic marker is the proportion of CD8 lymphocytes expressing the CD38 molecule, which is thought to be a measure of lymphocyte activation. Several investigators reported that the greater the proportion of $\mathrm{CD} 8$ cells expressing $\mathrm{CD} 38$, the more rapid the rate of progression to AIDS (WB2410, WB2422, WB2430).

(4) The degree of immune deficiency. The importance of a low CD4 lymphocyte count as an indicator of the risk of development of AIDS was confirmed in numerous presentations (eg MC3137, MC45. MC3148, MC3154, TUC40, TUB4, TUC99, WC44, THC52, THC57, WB2444, WC3015, TUC39). A poor in vitro lymphocyte response to mitogen stimulation was also shown to indicate a raised risk of AIDS (WB2441, WB2442). Cutaneous anergy was also confirmed as a predictive marker (WB2444, WC44, WB2441, WB2442). Of the early signs and symptoms of HIV infection, only oral thrush is apparently indicative of increased risk of AIDS independent of the CD4 count (WC44, THC52, WC99).

Identification of markers of progression is important so that we might be able to accurately predict who is likely to develop AIDS within a given time span, and to decide if and when to introduce certain treatments; the CD4 lymphocyte count alone is already being widely used as a predictor for this purpose. Markers of progression could also be of use as a surrogate endpoint (instead of the incidence of serious clinical disease) in large comparative clinical trials in HIV infection. An analysis of a study of zidovudine use from Australia attempted to ascertain the extent to which the change in CD4 lymphocyte count after treatment corresponds to the eventual clinical outcome (THB33). Patients in whom the CD4 count did not rise after the start of treatment were found to progress to AIDS and to death significantly more rapidly than those in whom the CD4 count rose, even if only temporarily. Those patients in whom the CD4 lymphocyte count remained above the pre-treatment count (median follow-up 104 weeks) experienced the most favourable prognosis.

Thus studies of markers of progression of HIV 
infection are making and, will continue to make, a valuable contribution to clinical decision making, to the efforts to identify suitable surrogate endpoints in clinical trials and to understanding the pathogenic process of HIV infection.

\section{Behaviour change-Graham Hart \\ Injecting drug users}

Crosier et al (MC48) reported an increase in HIV infection to $13 \%$ amongst a sample of street, agency and other social network recruited IDU's in London which was of some concern given the findings of an earlier study in groups attending and not attending needle-exchange in London and elsewhere (TUC54), which identified a reduction in reported risk behaviours over time (1989-90) with prevalences of $1.2 \%$ in attenders and $3.6 \%$ in non-attenders (possibly reflecting the higher level of risk behaviour in non-attenders). The rise in prevalence has occurred despite a massive increase in the availability of injecting equipment in the period 1987-90 resulting in only $21 \%$ of needle-exchange attenders reporting recent sharing behaviour, and overall reductions in risk behaviour in non-attenders (THC45). This suggests that, whilst there may be absolute reductions in risk behaviours, if the pool of infection is sufficiently large this will result in further infections in at risk individuals despite population based behavioral change.

Surveillance of risky behaviour and HIV infection in IDUs in the UK generally has been patchy until recently; Durante et al (WC3343) reported on combined behavioral and virological data in 1925 drug users, from over thirty drug agencies in England and Wales, and found an anti-HIV prevalence of only $1 \cdot 2 \%$. Recent sharing was limited to a minority of IDUs, although $32.5 \%$ of the total sample were anti-HBc positive, providing evidence of previous risk behaviour, and emphasising the need for continued monitoring of this population.

Once again we were reminded of differential risk behaviours according to drug of choice, with amphetamine users in North West England demonstrating increased sexual risk in terms of numbers of casual partners and generally low levels of condom use when compared with heroin users (TUD106). This was also the case amongst stimulant users in San Francisco and Seattle, where both sexual and injecting risks were higher than in opiate users, as was HIV infection ( $5 \%$ vs $1 \%$ ) (WC3308, WC3363). Sexual activity was reportedly at a high level among male IDUs using amphetamine in Sweden (WC3352).

A major change in risk behaviours was reported from Edinburgh drug users in the period 1986 to 1990, with few seroconversions and the suggestion that any future seroconversions are likely to be a consequence of heterosexual intercourse rather than of sharing as episodes of this behaviour fell dramatically in the period of study (MC3029).

Most controlled studies concerned with drug use and progression to AIDS have found no association between continuing drug injection and progression. However, drug of choice may affect progression; a study from New York found a reduction in CD4 count associated with continued injection of cocaine, suggesting that use of this drug is inadvisable for seropositive injectors (MC3156).

We often associate the USA with very high prevalences of HIV infection amongst drug users. In fact there is substantial variation across the country, some areas experiencing very low levels of HIV (THC46); including Ohio with a prevalence of $1.3 \%$ in 855 non-treatment IDUs (MC3213). Seropositivity in this study was associated with homelessness, travel to an HIV epicentre, daily cocaine injection and a history of sexually transmitted diseases, once more demonstrating the importance of risk factors additional to those of sharing injecting equipment and unprotected sexual intercourse in low prevalence areas.

A study from New York and Chicago, (MD4004) found that homeless IDUs were more likely than IDUs in stable living conditions to engage in high risk sexual and injecting behaviour. Access to condoms, syringes, bleach and other sterile drug paraphernalia was limited, and homeless IDUs were often denied access to these by other, more stable IDUs, as well as being less visible to welfare and criminal justice agencies.

More positively, a study from New York showed that there has been a move to incorporate avoidance of sharing into the city's drug culture. This was demonstrated by an investigation of early injecting behaviour and initiation to injecting. Although overall $56 \%$ of 1095 IDUs reported sharing on their first occasion of injecting, this fell from $65 \%$ of those initiated prior to 1978 to $34 \%$ initiated in the period 1986-89. However, those who commenced injecting drug use in this later period and had shared on their first occasion of injecting were more likely to have continued sharing practices, and introduced others to these, than those who had not been introduced to injecting by sharing (MD4057). This was essentially a study of the effect of peers on behaviour, and the use of peer mobilisation to effect behavioural change was investigated in another New York study which aimed to increase condom use through use of local ex-users in training sessions. Condom use did increase as a result of the intervention, suggesting the benefits of using current and ex-IDUs as part of prevention programmes (WD54). Peer support was also a significant factor in another report (WD4133).

In Australia, the low level of HIV infection $(<2 \%$; WC3330) may be as a result of the limited extent of sharing (MD4068). The European picture by way of 
contrast is extremely varied, with prevalences in EC countries as low as $2 \%$ (Greece) and as high as $75.5 \%$ (Spain) (MD4074).

In the USA there has been a major increase in crack cocaine use in recent years, (WC3294). Crack use is associated with HIV infection because of increased sexual activity, including exchanging sex for money and the drug itself (WC3304). In a study of HIV and syphilis infection in three groups of street recruited drug users and one group reporting no drug use in Miami, New York and San Francisco $(n=323)$ it was found that $15 \%$ of crack users, $50 \%$ of injectors, $35 \%$ of dual users and $8 \%$ of the nondrug using group were HIV positive; seropositivity for syphilis was $11 \%$ in crack users, $12 \%$ in injectors, $14 \%$ in dual users, and $4 \%$ in the remaining nondrug using street recruited population (THC49). The most sexually active groups, including people exchanging sex for money, were those who used crack; there was also a high prevalence of concurrent sexually transmitted disease in the crack users. This was also reported in other studies of women crack users compared with matched controls, with much increased risk of trichomonas infection and positive syphilis serology (WC3096). Sexually transmitted diseases have been shown to be present more frequently in drug using than non-drug using populations, emphasising the need for targeted STD services for this group (WC3348).

A number of posters reported on the positive effects of needle-exchange schemes in preventing HIV infections, particularly in those few American States where needle-exchange is not illegal (Connecticut, WC3286; Wasington State, WC3291, WC3362) and, in California, where it remains illegal, a high proportion of needle-exchange users reported safe needle-use (WC3295; THC99). Lundt, in Sweden, has also seen no epidemic of HIV infection in clients of its needle-exchange (WC3290). However, preliminary results of a study in Montreal showed there was an increase in HIV infection amongst attenders at a needle-exchange (WC3324).

Dolan et al (WC3321) reported on men and women who had recently (<three months) been released from prison, and found a prevalence of $10 \%$ in injecting drug users; a quarter of IDUs had continued injecting in prison in the $\mathrm{UK}$, and three quarters of these reported sharing. Amongst female injectors the prevalence was $15.5 \%$, and $3.4 \%$ of non-injecting heterosexual women were also positive. This was a large study by UK standards $(n=450)$ and may prove instrumental in changing Government policy with regard to HIV infection in prisons.

A study from Germany (MD4040) found increased levels of HIV infection and risk behaviours in male and female drug users who had engaged in prostitution as against those who had not; $30 \%$ of
IDUs with a history of prostitution were antibody positive vs $16 \%$ of those with no history of prostitution.

Sexual behaviour change was monitored in 5339 clients of 44 drug agencies in the USA in 1989-90, but it was found that only $30 \%$ reported using condoms in the year prior to interview. However, women and younger clients (15-24) reported higher levels of condom use, a trend that should be encouraged (WC3344). Condom use increased as a result of free provision in a drug treatment clinic in Seattle (WC3347).

\section{Behaviour change-gay men}

Unlike the UK, where recent reports suggest that young gay men have adopted and maintained safer sex behaviours, in the USA the level of risk among men in the age range 18-27 years is high, with a third of men (in one Californian study) reporting unprotected anal intercourse in the last two months (MC101). A Canadian study found that seronegatives who persisted in unprotected anal intercourse with casual partners were more likely to be younger, of lower socioeconomic status, to smoke and drink more heavily, and were more likely to use nitrite inhalants than seronegative controls (WC3003). The relationship between recreational drug use and unsafe sex was highlighted in two studies (MD4071, WC3009). Younger gay men in San Francisco reported more unprotected anal sex than older men; however, much of this was accounted for by being in a committed relationship (WC3004). Nevertheless, the prevalence of HIV infection was very high in young men in San Francisco County as compared with other Bay Area Counties, as seen in STD clinic attenders (WC3010).

There were many more posters this year concerned with bisexual men. One behavioral study found that, compared with exclusively homosexual men, bisexual males were more likely to report unprotected intercourse, with both male and female partners (MD4014). There were differences between black and white bisexual men reported in another poster, showing that whites were more likely to meet male partners in anonymous settings (bathhouses etc) whereas blacks met partners more frequently in bars. Blacks were less likely to have a current female partner, but more likely to report more episodes of unprotected anal intercourse with male partners (MD4042).

Two studies (WD52, WD4139) criticised the use of the term "relapse" on the grounds that a change from safe to unsafe sexual behaviour in any given period of time does not necessarily mean a loss of control over sexual behaviour or a reverting back to old ways. Neither paper found any difference between the so-called "relapse" groups and other subpopulations of gay men, except that they were usually 
engaging in unsafe sex in the context of a primary relationship. Relapse studies generally are based on cohorts, and one paper investigated the extent to which such cohorts are biased towards men whose behaviour is "safer" anyway. The authors found that, at each wave of a longitudinal study, study participants became over-representative of low level risk, and therefore rapidly declining seroconversion rates in these groups may not necessarily reflect infection in the community as a whole (WC95).

\section{Neuroscience of HIV infection-Hadi Manji}

Headaches, lumbar punctures and HIV infection

Burak (MB2000) in a retrospective study, found that in patients with headache, fever or altered mental status, the diagnostic yield of lumbar puncture was $24 \%$. Headache, in particular, was present in $86 \%$ of this group. However, serious intracranial pathology could present without headache. A CD4 count of less than 200 also increased the likelihood of the lumbar puncture revealing a definite, potentially treatable disorder. Trenkwalder (MB2133), looking at recent onset severe headaches found $35 \%$ of patients were diagnosed as having opportunistic infections (OI) or tumours, $53 \%$ tension like headaches and $12 \%$ had other classifiable headaches such as migraine. However, it was not possible to differentiate between these headaches and they concluded that recent onset headaches need a full clinical assessment and consideration of $\mathrm{CT}$ and lumbar puncture.

\section{Toxoplasmosis}

A London clinic study showed that $23 \%$ of 233 AIDS patients were $T$. gondii seropositive and the incidence of toxoplasma encephalitis was low at $4.7 \%$ (WB2349).

Katalama presented the preliminary results of the European Network of Treatment of AIDS (ENTA) trial of oral Pyrimethamine/Clindamycin versus oral Pyrimethamine/Sulphadiazine (WB30). The results were similar to those of the Californian Collaborative Trials Group presented last year, suggesting that the two drugs were similar in efficacy. However, drug intolerance was higher in the Sulphadiazine group.

Finally, looking to the future Masur (WB31) reported on $566 \mathrm{C} 80$, a hydroxynaphtoquinolone, which has been shown to destroy toxoplasma cysts in vitro and in vivo which in theory would make maintenance therapy unnecessary. It is also effective against Pneumocystis carinii.

\section{Cryptococcal meningitis (CM)}

Waskin (MB2007) looking at survival trends in CM found a death rate in the acute phase of $26 \%$. At the satellite symposium, the updated version of the ACTG/MSG trial comparing fluconazole and amphoterin B was presented. As at last year's meeting, there was still some wariness with the use of fluconazole because of the longer time taken to sterilise the CSF and the higher early death rate. There was no such doubt when considering the use of fluconazole $200 \mathrm{mg}$ daily for maintenance therapy. At present, therefore amphoterin $B$ should perhaps still be used in the so called higher risk group ie those with altered mental status, positive india ink staining of the CSF, hyponatraemia and crytococcal CSF titres greater than 10,000. The issue of any beneficial effects of 5 flucytosine in AIDS associated CM is still not resolved and is under trial.

Carluccio (WB2301) looked at the question of whether the use of anticandidal drugs, fluconazole and ketoconazole, altered the natural history of CM. Patients on these drugs tended to have a later presentation (with a consequent worse outcome), had blunting of their physical signs and had a high rate of negative CSF cultures. However, CSF antigen was always positive. It should be noted that the numbers in this study were small.

\section{Cytomegalovirus (CMV) and the nervous system}

In the early stages of the epidemic CMV retinitis was considered a pre terminal event. Fisher (WB2332) looking at the survival and visual outcome during long term therapy found a mean survival after a diagnosis of CMV retinitis of 17.6 months. Spector (MB86) presented the results from the ACTG 071 study comparing immediate versus delayed treatment of peripheral retinitis with ganciclovir. The median time to progression was significantly shorter in the treated group ( 50.5 days versus 15 days). It was suggested that all patients with a low CD4 count should undergo ophthalmological screening at regular intervals to pick up any cases of early CMV retinitis. However, the study did not answer the question as to whether, in the long term, the deferred treatment group fared less well. A caveat with this study was their definition of "peripheral"-lesions greater than $1500 \mu \mathrm{m}$ from the optic disc or greater than $3000 \mu \mathrm{m}$ from the fovea.

The clinical presentation of CMV encephalitis is still far from clear. Berman (WB2366) described two autopsy proven cases of fulminant encephalitis in patients on ganciclovir for retinitis. Their clinical presentation was characterised by a rapid change in mental status. Neuroradiological and CSF examinations were unhelpful in the diagnosis. This may be a diagnosis worth considering in a rapidly deteriorating patient for which no other cause can be found.

Progressive multifocal leukoencephalopathy (PML) The prognosis for PML remains poor. Levy (Padua, p10) presented data on 13 cases which were diagnosed early by biopsy. The mean survival was 2.5 months, in spite of aggressive symptomatic therapy which included zidovudine but no specific anti JC 
virus therapy. Britten (Padua, p23) described the use of intrathecal cytosine arabinoside with improvement in 5 of 10 cases. Portiges (MB2037) presented three cases treated with systemic cytosine arabinoside. A multicentre controlled study is under way to study the efficacy of this drug in PML.

\section{Myopathy, zidovudine and HIV}

Dalakas who claims that zidovudine-associated myopathy is characterised by the presence of ragged red fibres and abnormal mitochondria backed this using rat models (WA63) and muscle culture techniques. A study by Gherardi (Padua, p109), has supported some of Dalakas' findings. However, Simpson (Padua, p57) has found no clinical or histological differences between the zidovudine exposed and non exposed group. A useful tip from him was that the iliopsoas muscle is the most sensitive in which to pick up myopathic changes on EMG.

\section{Neuropathological studies}

Gray (Padua, p35) described the neuropathological findings from the brains of asymptomatic HIV infected individuals who had died from causes unrelated to their HIV status. The 11 cases showed evidence of myelin pallor, astrocytosis and microglial proliferation. They were unable to find evidence of multinucleated giant cells or demonstate any HIV antigens.

There is increasing evidence of HIV affecting both grey and white matter: Sinclair (Padua, p58) showed the presence of HIV proviral sequences in both grey and white matter using PCR techniques. Up to $38 \%$ neuronal loss in the frontal cortex was found by Everall (MB30) studying brains from the Medical Research Council Central Aids Brain Bank. This neuronal loss was unrelated to the presence or absence of HIV encephalitis.

There were numerous posters describing the benefits of zidovudine in HIV encephalopathy. However, to date most of these have been anecdotal or open uncontrolled trials. One confounding variable difficult to overcome has been an improvement of cognitive function due to any concomitant symptomatic improvement.

Vago (WB28) presented autopsy data using the presence of multinucleated giant cells as a marker for HIV encephalitis. There was a significant reduction in the zidovudine treated as opposed to the untreated group. Furthermore, the least number of multinucleated giant cells was seen in the group who had been on treatment for 6 to 12 months. In patients who had been on zidovudine for between 12 to 30 months, the frequency of this marker increased. The numbers, however, in this part of the study were small. This concurs with Portiges' retrospective study which suggested a decline in the incidence of HIV encephalopathy since the introduction of zidovudine in 1987.
Clinical aspects I-Annemiek de Ruiter

Pneumocystis carinii pneumonia (PCP)

There were a large number of studies on the use of nebulised pentamidine as a prophylactic agent for primary and secondary PCP. The doses used in these studies ranged from $60 \mathrm{mg}$ twice a week (WB2244) to $600 \mathrm{mg}$ weekly (WB2230) with probably the most frequently reported doses being $60 \mathrm{mg}$ every 2 weeks (MB2173, WB2191, WB2209) or $300 \mathrm{mg}$ every month (THB41, MB2160, WB2245).

Four studies with a total of 364 patients that did compare various combinations of co-trimoxazole, pentamidine and dapsone with or without pyrimethamine at a variety of doses, felt that all these agents were equally effective for both primary and secondary PCP prophylaxis although all four reported the highest incidence of side effects with co-trimoxazole (WB2241, WB2245, WB2247, WB2224). However, a larger study from California of 211 patients on either co-trimoxazole $960 \mathrm{mg} 3$ times a week, dapsone $50 \mathrm{mg}$ a day or aerosolised pentamidine $300 \mathrm{mg}$ every month for primary, secondary or tertiary PCP prophylaxis, followed over a 2 year period showed that there was one episode of PCP per 981 patient-months in the co-trimoxazole group compared to one episode in 87 and 69 patientmonths in the dapsone and pentamidine groups respectively. They concluded that co-trimoxazole was the most effective drug but did again result in the highest incidence of side effects (MB2160).

A study from Australia of 171 patients receiving either co-trimoxazole $960 \mathrm{mg}$ bd twice a week or aerosolised pentamidine $300 \mathrm{mg}$ every 4 weeks for secondary PCP prophylaxis showed a statistically significant higher relapse rate in the group receiving aerosolised pentamidine (WB2246).

The optimum dose of co-trimoxazole remains to be established. An American study followed 128 patients receiving co-trimoxazole $960 \mathrm{mg}$ bd or 960 mg 3 times a week for primary or secondary PCP prophylaxis. The three times a week dose was associated with less side effects and was equally effective in preventing PCP (WB2199).

Several studies looked at dapsone and fansidar at varying doses and found them to be effective (WB2207, WB2208, WB2181, WB2197, WB2242). A study of 96 patients receiving either dapsone 100 $\mathrm{mg}$ twice a week or aerosolised pentamidine $400 \mathrm{mg}$ every month concluded that there was no apparent difference in efficacy between the 2 agents (WB2228).

A question that was raised by several studies was whether prophylactic agents should be used that protected against both PCP and cerebral toxoplasmosis. One study from Germany noted a high incidence of cerebral toxoplasmosis in patients on secondary PCP prophylaxis with aerosolised pentamidine (WB2189). A study from the same centre followed this up by comparing fansidar one tablet twice a week with $300 \mathrm{mg}$ of aerosolised 
pentamidine looking separately at primary and secondary PCP prophylaxis. In this group of 147, fansidar and pentamidine appeared equally effective for primary and secondary PCP prophylaxis but cerebral toxoplasmosis occurred significantly more frequently in the patients receiving aerosolised pentamidine. They concluded that fansider may be better than aerosolised pentamidine for secondary PCP prophylaxis in areas with a high toxoplasma seroprevalence (WB2212).

A study from Switzerland looked at this question from a different angle by comparing a group of patients on sulphadiazine and pyrimethamine for toxoplasma maintenance therapy with a control group. No patient in either group was on standard PCP prophylaxis or had a history of PCP. The incidence of PCP was significantly lower in the group on toxoplasma maintenance therapy and they concluded that maintenance therapy of cerebral toxoplasmosis may also protect against PCP (WB2218).

Regarding the treatment of PCP several studies confirmed the efficacy of aerosolised pentamidine and co-trimoxazole. In addition, an American group studied the use of oral clindamycin and primaquine for the treatment of mild to moderate PCP. Thirty out of 38 patients were successfully treated with five patients withdrawing due to side effects, primarily rash, and of the three treatment failures one patient was known to be non compliant (THB42). Finally an experimental oral drug $566 \mathrm{C} 80$ successfully treated 27 out of 34 patients with PCP (WB2239).

\section{Cytomegalovirus pneumonitis}

A study from the Royal Free Hospital looked at nine patients with respiratory symptoms in whom bronchoalveolar lavage CMV was identified as the only infectious agent. In seven patients with a CD4 count of less than $0.1 \times 10^{9} / 1$ the symptoms were mild and no specific anti CMV therapy was necessary. However, two patients with a CD4 count of 0.23 and $0.27 \times 10^{9} / 1$ had a severe illness requiring anti CMV therapy and a period of ventilatory support. In both these patients the CD4 count later rose significantly and there was evidence that this may have been part of a seroconversion illness (THB40).

\section{Haematology}

A cohort study of 174 patients with HIV related thrombocytopenia was presented by a group from France. Most were intravenous drug users and all had a platelet count of less than $50 \times 10^{9} / 1$. Sixty percent had a transient response to steroids. High dose intravenous immunoglobulin was effective in $84 \%$ of patients but again the effect was transient. Zidovudine at doses varying from 500 to $1000 \mathrm{mg}$ a day resulted in an initial response in $\mathbf{7 0 \%}$ of 84 patients which was sustained on therapy in $53 \%$, the best responses being seen in the highest dose of zidovudine and in patients with a low $\mathrm{CD} 4$ count. Sixty-two underwent splenectomy and $85 \%$ showed a sustained response. Splenectomy did not have any effect on survival which was related to the CDC stage of the patient (WB82).

An Italian study examined the long term efficacy of zidovudine on 125 patients divided into two groups, one with a platelet count of less than $50 \times 10^{9} / 1$ (Group A) and the second with a platelet count between 50 and $100 \times 10^{9} / 1$ (Group B). At the start of therapy the mean CD4 count was higher in the group with the lower platelet count. During the first 3 months of therapy $67 \%$ of patients in Group A responded compared to $86 \%$ in Group B. All increased platelet counts were sustained over 18 months while in most patients the CD4 count rose then fell. In conclusion they showed that zidovudine increased the platelet count in the majority of patients and that this rise was sustained despite a deterioration in the CD4 count (WB83). Both groups concluded that thrombocytopenia was of no prognostic significance.

\section{Non-Hodgkin's lymphoma}

Several studies addressed the question of the possible relationship between zidovudine and non-Hodgkin's lymphoma. In one study eight of 55 patients on zidovudine developed non-Hodgkin's lymphoma. All had a CD4 count of less than $50 / \mathrm{mm}^{3}$. They calculated that the risk of developing non-Hodgkin's lymphoma was $29 \%$ at 36 months (TUB4). A second study showed a lower incidence with 24 out of 1028 patients on zidovudine developing non-Hodgkin's lymphoma after 2 years of treatment (TUB81).

A study from Sweden reported on the development of a malignant lymphoma very similar to Human HIV-associated lymphoma in 10 out of 24 Cynomolgus monkeys infected with Simian Immunodeficiency virus, none of which had received zidovudine (TUA8).

The overall conclusion was that there is no evidence that zidovudine causes non-Hodgkin's lymphoma and that prolonged survival in immunosuppressed patients is the more likely cause.

Regarding the epidemiology of non-Hodgkin's lymphoma an Italian study analysed data from 8068 AIDS cases reported to the Italian National AIDS registy by November 1990 and found 320 cases of non-Hodgkin's lymphoma. Primary cerebral lymphoma occurred three times more commonly in intravenous drug users compared with homosexual men whilst the latter were twice as likely to develop systemic lymphoma. In addition, whilst the incidence of non-Hodgkin's lymphoma had decreased in intravenous drug users between 1985 and 1990 it had shown a slight increase in homosexual men (TUC102). 
Genital neoplasia

Several studies reported a high prevalence of Cervical Intraepithelial Neoplasia (CIN) and high risk HPV types in HIV positive women (WB2376, MB2403, MB2259, MB2408, WC3136).

A study from New York looked at the CD4 counts and cervical smears of $44 \mathrm{HIV}$ positive women. The prevalence of CIN was higher in those with CD4 counts below $0.2 \times 10^{9} / 1$ and in addition all five women with CIN III were in this group (MC97). A study from Malawi of $64 \mathrm{HIV}$ positive and 95 seronegative women noted a higher prevalence of CIN in the HIV positive group but no difference in the prevalence of $\mathrm{CIN}$ when comparing women with CD4 counts above and below $0.4 \times 10^{9} / 1$ (MC98). A study from Italy looking at the natural history of HPV and CIN in both HIV positive and negative women suggested that progression rates were higher in HIV positive women (MB2425).

Two studies noted a high prevalence of anal intraepithelial neoplasia (AIN) on histology in both HIV positive and HIV negative men with anal condylomata. Severe dysplasia (AIN III) was more common in the HIV positive group (MB2221, TUB86). At present the natural history of AIN is unknown, but it is possible that as patients continue to live longer in an immunosuppressed state that this may become a problem.

\section{Clinical aspects II-B Ian McGowan \\ Kaposi's sarcoma}

Martinez-Maza et al (TUA5) showed that AIDS' Kaposi's sarcoma (KS) cells when exposed to HIVTAT produced increased levels of IL-6 mRNA and IL-6. The authors postulated that continued exposure to high levels of IL-6 might cause proliferation within AIDS' KS cell lines.

Therapy for KS remains difficult, as does assessment of drug trials. The studies presented were small, with poorly defined populations and vague end points. Tabah et al (WB2374) selected a relatively healthy group of patients with $\mathrm{KS}$ (no previous history of opportunistic infection, and CD4 count $>150$ ). Patients were randomised to one of three doses of interferon alpha $(10,15$, or $20 \mathrm{MIU} /$ day). 20/24 evaluable patients showed a response, irrespective of dose. Sloand et al (MB2178) treated 14 patients with pulmonary $\mathrm{KS}$ with daunorubicin, bleomycin, vinblastine, vincristine, actinomycin and dacarbazine. This regime was well tolerated and mean survival was increased from 3/12 (in historical controls) to $8 / 12$.

\section{Mycobacterium Avium-Intercellulare (MAI)}

Rapid diagnosis of MAI is not routinely available, but Bollet et al (WB2256) described a polymerase chain reaction technique (PCR), for MAI which produced results in 4 hours.
Tripathy et al (WB2314) showed that $89.8 \%$ of hospitalised patients with MAI were HIV positive. Survival following diagnosis is poor, in Smith et al's study of 73 patients, 33\% died within 1 month, and the 6 month survival was only $11 \%$ (WB2310).

Jorup-Ronstrom et al (MB2365) reported the use of amikacin, ethambutol, and rifabutin, in 31 patients. Seventy-one percent of patients improved (fall in temperature, reduced diarrhoea and increased quality of life). New agents showing some promise include liposome encapsulated gentamicin (WB2341) clarithromycin (WB2358) and sparfloxacin (WA1051).

\section{Cardiovascular}

Information presented at Florence suggested that between $17 \%$ and $61 \%$ of HIV positive patients have evidence of heart disease (MB2362, MB2374, MB2401). Clinical symptoms are uncommon (MB2401), and most abnormalities are detected by Doppler and/or echo-cardiography. Ventricular dysfunction and dilated cardiomyopathy were the most common changes seen.

\section{Gastroenterology}

Fleming et al (TUB27) demonstrated that HIV can directly infect cells of the lamina propria in vitro and this finding may be important in the pathogenesis of HIV enteropathy. Zeitz et al (WB90) showed that malabsorption (defined by an abnormal $\mathrm{H}_{2}$ exhalation, xylose tolerance, or Schilling test) occurs at all stages of HIV infection independently of diarrhoea or opportunistic infection.

Non opportunistic enteric infections may have an altered natural history in HIV immunosupressed patients. Delcroix et al (MB2261) reviewed 20 patients with campylobacter enteritis, and noted a high prevalence of resistant strains, and copathogens.

\section{Nutrition}

Scevola et al (WB2169) looked at 404 Italian patients and found that the prevalence of malnutrition was $36.2 \%$ in asymptomatic patients rising to $88.4 \%$ in AIDS patients. Sixty percent of AIDS patients in the USA use nutritional supplements (NS) and Beall et al (MB2194) carried out 7-day food records combined with anthropometric assessment and showed that AIDS patients using NS fared no better than those who did not. Both enteral (MB2241) and parenteral therapy (MB2428) may have some benefit.

Four centres reported on the use of megestrol acetate (MA) in AIDS associated anorexia and weight loss (MB2198, MB2263, MB2233, WB2392). The largest study (WB2392) had 176 evaluable patients, and $67 \%$ of patients on $800 \mathrm{mg}$ gained in excess of $2 \mathrm{~kg}$, compared with $16 \%$ on placebo. 


\section{Thalidomide}

Gehanno et al (MB2225) reported on the use of thalidomide in 22 patients with severe apthoid ulceration. Nine had no treatment and the lesions healed within 2 to 9 months. In patients receiving 100 $\mathrm{mg}$ thalidomide qds for 5-15 days, pain resolved within 1-4 days, with healing occurring within 5 days. No comment was made about side effects of thalidomide.

\section{Cryptosporidium}

Using a differentiated human enterocyte cell line Marshall et al (WB2269) showed that hyperimmune bovine colostrum could inhibit the development of Cryptosporidium parvum. This system, if validated may be beneficial in screening potential agents for anti-cryptosporidial activity. Paromomycin $(500 \mathrm{mg}$ qds 4-31 days) was used to treat 22 patients in an uncontrolled study and showed some efficacy (MB2270).

\section{Candidiasis}

A number of trials compared the use of conventional agents in the treatment of oral and oesophageal candidiasis. Itraconazole ( $200 \mathrm{mg}$ od 7 days) gave a $96 \%$ day 14 cure rate compared with $90 \%$ with fluconazole (50 mg od 7 days) (MB2201). Fluconazole (100 mg qds 2-8 weeks) had a cure rate of $91 \%$ compared with $52 \%$ with Ketoconazole ( $200 \mathrm{mg}$ qds 2-8 weeks) (WB2264). Leen et al (WB2317) documented resistant candidiasis in three patients who had received fluconazole for over 1 year. Treatment was given with intravenous amphotericin and five flucytosine.

\section{Microsporidium}

Using electron and light microscopic examination of intestinal biopsies in 48 AIDS patients, Bernard et al (MB2216) found the prevalence of microsporidium to be $10.5 \%$. Small studies with albendazole $(400 \mathrm{mg}$ bd 4-6 weeks) (WB2265), and metronidazole (500 mg tds mean 12.3 days) (WB2267), showed some efficacy in microsporidial diarrhoea.

\section{Cytomegalovirus (CMV)}

Salberger et al described the detection of CMV-antigen by immunohistochemistry, using a variety of monoclonal reagents in 238 biopsies. They concluded that the presence of intranuclear early antigen is specific for active infection (WB2316). Dietrich et al (WB2293) treated 14 patients with biopsy proven CMV GI disease ( 7 oesophagitis, 7 colitis) with foscarnet. All patients had relapsed on ganciclovir. Improvement was seen in 11/14 (improved endoscopic appearance, decreased symptoms). However, 3/7 patients developed oesophageal strictures requiring dilatation and survival was not improved. Hawkins et al used foscarnet as first line treatment (WB2262). Patients received 3 weeks of therapy. Fifteen patients (18 episodes) were treated with oesophageal, and 22 with colonic disease $(27$ episodes). In the oesophageal group 15/18 episodes responded within 14 days with complete loss of symptoms. In the colitis group $11 / 18$ patients being treated for their first episode went into remission.

\section{AZT absorption}

As clinicians move towards lower dosing regimes for antiretroviral therapy, it is important that the individuals are absorbing sufficient quantities of drug for clinical efficacy. Three studies looked at AZT absorption. Kapembrwa et al showed a $32 \%$ reduction in eight patients with small intestinal disease, compared with five post Pneumocystis carinii controls (MB2264). In contrast Taburet et al (MB2275) could detect no fall in bioavailability of AZT in patients with chronic diarrhoea, although the number of patients was not specified. Sahai et al (WB2079) showed no reduction following a protein meal.

\section{Hepatitis}

Errera et al looking at 710 Italian, mainly IDUs, patients found an overall prevalence of anti-HCV to be $70 \%$ in HIV positive patients, compared with $51 \%$ in HIV negative patients (MB2205). These results were obtained with first generation tests which have been associated with high false positive rates. Fegueux et al in France found anti-HCV in $48 \%$ of IDUs, $13.3 \%$ of gay men, and $5.6 \%$ of heterosexuals (MB2209). Chirianni et al in Naples (WB87) commented on a declining prevalence of anti-HCV as CD4 count falls, analogous to the loss of anti HBs in HIV positive patients. Cohen reviewed the HBV status of 96 patients attending a HIV outpatient clinic, and noted that $43 \%$ might benefit from vaccination, he also noted a sub group of 24 patients who had lost anti HBs. Twenty of these patients were IDUs (WB91).

\section{Liver biopsy}

Pol reviewed the role of liver biopsy in AIDS patients referred to a liver unit. Of 12 biopsies performed, seven lead to a diagnosis within 1-3 days, most commonly MAI or M. tuberculosis (MB2213). In a larger study from Spain (MB2384) 82 biopsies were performed. The biopsies were divided into two groups

A) Fever with clinical suspicion of systemic infiltrative disease,

B) Hepatomegaly $+1-$ abnormal liver function tests.

In group A (39) 16 patients had tuberculosis and 4 had evidence of CMV. In group B (43) 16 patients had evidence of chronic active hepatitis. 
Clinical aspects III-Melinda Tenant-Flowers

Management of paediatric HIV

Mofenson et al (TUB96) gave intravenous immunoglobulin prophylaxis for severe bacterial infections to 372 children in a placebo controlled trial and demonstrated a significant increase in the infection free survival time in those with a CD4 count of greater than $200 / \mathrm{mm}^{3}$. However, there was no difference in mortality between the two groups. Simonds et al (WC35) studied 2,327 perinatal AIDS cases $916(39 \%)$ of whom had PCP. Seventy percent of cases occurred in infants below the age of 1 year and had an increased risk of death in the 2 month period after their PCP diagnosis. This indicates that PCP prophylaxis should be commenced immediately after treatment for PCP and perhaps, at birth for all children at risk of HIV infection.

\section{Needlestick injuries}

Henry et al (MD58) looked at compliance with universal precautions in the emergency departments of two Minneapolis hospitals. They found that observed compliance was significantly less than self reported with regard to the use of protective clothing, recapping needles and glove injuries. Glove penetration resulted in blood contamination of the operative's hands in $7 \%$ of cases. Bell et al (MD59) estimated the risk of HIV and hepatitis B transmission to patients from surgeons involved in 1,382 procedures to be between 0.0002 to $0.002 \%$ for HIV and for hepatitis $B$ between 0.024 and $0.24 \%$. Ippolito et al (MD61) looked at occupational risk of HIV transmission in 30 hospitals. They found that nurses were the most at-risk group, reporting $64 \%$ of exposures and that $57 \%$ of exposures took place on the wards mostly while phlebotomy was being performed. Jaffe et al (THD110) reported on HIV transfer to patients during dental care. Six patients of a dentist with AIDS were subsequently found to be HIV antibody positive; two had no other risk factors for HIV and in three viral analyses showed that their HIV was similar to that of the dentist's. However, infection control procedures were not scrupulously observed in this practice and the exact route of transmission remains unclear.

\section{Anti-retroviral therapy}

Aboulker et al (WB2117) presented an update on the MRC INSERM Concorde Trial of AZT for asymptomatic HIV infection. One-thousand-andseventeen persons have been recruited so far. There have been 35 deaths, 80 progressors, 54 lost to followup, 165 have stopped capsules but continue followup and 185 have stopped capsules and started AZT treatment because of a falling CD4 count. The Data and Safety Monitoring Board will be reviewing the interim analysis in October 1991. The largest study of AZT administration to women came from Houle et al (WB2085). Two hundred and eighty nine women received AZT at doses between 200-1200 mg per day. Anaemia occurred in $9.5 \%$ versus $7.7 \%$ in what was said to be a comparable group of men. The issue of whether AZT is safe to give in pregnancy was explored by Ferrazin et al (MC3023). Seven women took AZT from 16-30 weeks festation; there were no foetal malformations or side effects noted.

Creagh-Kirk et al (TUB93) studied AZT administration in 540 children. The drug was well tolerated in $92 \%$ with increased weight gain and stature. Elia et al (TUB98) demonstrated favourable surrogate marker changes in 167 symptomatic children given AZT. Butler et al (TUB94) showed that ddI given to 89 children, 50 of whom had previously received AZT resulted in 14 severe side effects including six with pancreatitis. Easterbrock et al (THB37) demonstrated no racial or ethnic differences in response to AZT treatment in 1,025 patients provided the drug was administered at comparable times in the course of their disease.

Aboulker et al (WB2096) presented preliminary data on the MRC/INSERM Alpha Trial of ddI in AZT intolerant patients. One-thousand-and-twohundred people have been recruited although only 16 have been randomised to the arm where they might receive placebo. They mainly have AIDS or ARC and in $78 \%$ of cases have previously received AZT. To date there have been 134 deaths, 74 progressions, 71 have stopped therapy, 147 have had their dose modified and there have been five cases of pancreatitis. Nelson et al (WB2111) presented data on toxicity of ddI in a phase $2 / 3$ study of 153 patients. There were six cases of pancreatitis of whom two died, and newly reported adverse events include diabetes mellitus and Raynaud's syndrome. Forty-nine deaths occurred in total, three of which were sudden in patients suffering from MAI.

The rationale for combination therapy is that it may improve efficacy, decrease toxicity and prevent or delay the emergence of resistance. Collier et al (TUB2) presented the preliminary results of the ACG 010 trial of combination AZT (150-600 mg/ day) and ddI (90-300 mg/day) which has so far been given to 55 patients for up to 24 weeks. It has been well tolerated with $32 \%$ decreasing their dose or stopping therapy. Treatment resulted in decreased beta 2 microglobulin levels and in the group receiving the highest daily dose of AZT (600 mg/day) the CD4 count remained elevated for up to 24 weeks. Frissen (THB35) presented a combination trial of $\mathrm{AZT}$ and interferon alpha. Forty patients either received AZT $1000 \mathrm{mg}$ a day or AZT 500 milligrams per day plus interferon $3 \mathrm{MU} \times 3 /$ week. There was no difference in p24 Ag between the two groups at 24 weeks. Pluda et al (THB84) presented a three agent study where weekly cycles of AZT plus acyclovir/ ddI/ddC were given to 21 patients for up to 18 
months. The regimen was well tolerated, and there was an initial increase in CD4 count and fall in p24 antigen; however, this was not sustained beyond week 52 . Therefore, combination therapies studied so far have been well tolerated but these are only small studies and clinical efficacy remains to be demonstrated.

Several new approaches have undergone small, phase 1 studies and show no toxicity. These include bone marrow transplant (THB81, THB82) CD8 infusions (THB83), neutralising antibody infusions (THB85) and TIBO derivative R82913, (THB86). 